\title{
CHECKLIST DE CIRURGIA SEGURA: CONHECIMENTO E UTILIZAÇÃO DO INSTRUMENTO NA PERSPECTIVA DOS TÉCNICOS DE ENFERMAGEM
}

\section{CHECKLIST FOR SAFE SURGERY: KNOWLEDGE AND USE OF THE INSTRUMENT FROM THE PERSPECTIVE OF NURSE TECHNICIANS}

\section{CHECKLIST DE CIRUGÍA SEGURA: CONOCIMIENTO Y USO DE LA HERRAMIENTA EN LA PERSPECTIVA DE LOS TÉCNICOS DE ENFERMERÍA}

Núbia Conceição Santos Ferreira ${ }^{1}$, Luciane Ribeiro $^{2}$, Érica Toledo Mendonça ${ }^{3}$, Marilane Oliveira Fani Amaro ${ }^{4}$

\begin{abstract}
RESUMO
Objetivo: compreender o conhecimento e práticas dos técnicos de enfermagem sobre a aplicação do checklist de cirurgia segura em um hospital de ensino. Método: pesquisa qualitativa que contou com a participação de dez técnicos de enfermagem que atuam como circulantes de sala no Centro Cirúrgico. A coleta de dados foi realizada por meio de entrevista com roteiro semiestruturado e os dados foram submetidos à Análise de Conteúdo. Resultados: dos depoimentos dos técnicos de enfermagem emergiram três categorias: "Cirurgia segura na concepção dos técnicos de enfermagem", "A prevenção do erro em centro cirúrgico como sinônimo de identificação do paciente e atenção aos parâmetros clínicos" e "Compreendendo o uso do checklist de cirurgia segura: dissonâncias teórico-práticas". Conclusão: os técnicos de enfermagem demonstraram não terem conhecimento claro e fundamentado teoricamente sobre os aspectos que envolvem a segurança cirúrgica, não reconhecem o instrumento como ferramenta de prevenção/redução dos erros comumente ocorridos em centro cirúrgico e não sabem utilizá-la de maneira adequada.
\end{abstract}

Descritores: Lista de checagem; Time out na assistência à saúde; Segurança do paciente; Enfermagem de centro cirúrgico.

\begin{abstract}
Objective: to understand the knowledge and practices of nurse technicians on the application of safe surgery checklist in a teaching hospital. Method: Qualitative research that counted on the participation of ten nurse technicians who act as circulating of room in the Surgical Center. An interview with semi-structured script was applied and the data was submitted to Content Analysis. Results: Three categories emerged from the testimonies of the nurse technicians: 'Safe surgery in the conception of nurse technicians", "The prevention of surgical center error as a synonym of patient identification and attention to clinical parameters" and "Understanding the use of the safe surgery checklist: theoretical-practical dissonances". Conclusion: The nurse technicians demonstrated that they do not have clear knowledge and, theoretically based on the aspects that involve surgical safety, they do not recognize the instrument as a tool for prevention/reduction of errors that commonly occur in Surgical Centers and do not know how to use it properly.
\end{abstract}

Descriptors: Checklist; Time Out, Healthcare; Patient safety; Operating room nurse.

\section{RESUMEN}

Objetivo: Comprender el conocimiento y las prácticas de los técnicos de enfermería sobre la aplicación del checklist de cirugía segura en un hospital de enseñanza. Método: Investigación cualitativa que contó con la participación de diez técnicos de enfermería que actúan como circulantes de sala en el Centro Quirúrgico. La recolección de datos fue realizada a través de una entrevista con un guion semiestructurado y los datos fueron sometidos al Análisis de Contenido. Resultados: De los testimonios de los técnicos de enfermería surgieron tres categorías: "Cirugía segura en la concepción de los técnicos de enfermería", "La prevención del error en centro quirúrgico como sinónimo de identificación del paciente y atención a los parámetros clínicos" y "Comprender el uso del checklist de cirugía segura: disonancias teórico-prácticas". Conclusión: los técnicos de enfermería no demosntraram tener un conocimiento claro y fundamentado teoricamente sobre los aspectos relacionados con la seguridad quirúrgica, no reconocen el instrumento como una herramienta de prevención/reducción de los errores que ocurren comúnmente en el centro quirúrgico y no saben utilizarla de manera adecuada.

Descriptores: Lista de verificación; Pausa de seguridad en la atención a la salud; Seguridad del paciente; Enfermería de quirófano.

${ }^{1}$ Graduada em Enfermagem. Mestranda em Ciências da saúde pela Universidade Federal de Viçosa. ${ }^{2}$ Graduada em Enfermagem. Doutoranda em Saúde Coletiva pela Universidade Federal de Juiz de Fora. Docente na Universidade Federal de Viçosa. ${ }^{3}$ Graduada em Enfermagem. Doutora em Ciência da Nutrição pela Universidade Federal de Viçosa. Docente na Universidade Federal de Viçosa. ${ }^{4}$ Graduada em Enfermagem. Doutora em Biologia Celular e Estrutural pela Universidade Federal de Viçosa. Docente na Universidade Federal de Viçosa.

\section{Como citar este artigo:}

Ferreira NCS, Ribeiro L, Mendonça ET, et al. Checklist de Cirurgia Segura: Conhecimento e Utilização do Instrumento na Perspectiva dos Técnicos de Enfermagem. Revista de Enfermagem do Centro Oeste Mineiro. 2019;9:e2608. [Access Available in: . DOI: http://dx.doi.org/10.19175/recom.v9i0.2608 


\section{INTRODUÇÃO}

Atualmente, o interesse pela segurança do paciente nos serviços de assistência à saúde tem sido impulsionado pelos resultados de inúmeras pesquisas que evidenciam a extensão e o impacto do cuidado inseguro. A segurança do paciente é entendida como a redução, a um nível mínimo aceitável, do risco de dano desnecessário associado à atenção à saúde e é um dos critérios básicos para se garantir a qualidade da assistência ${ }^{(1)}$.

No contexto da segurança do paciente destacam-se os Eventos Adversos (EA) ocasionados em decorrência do erro cometido por profissionais de saúde. De acordo com a Organização Mundial de Saúde (OMS), entendese por EA todo incidente ou circunstância evitável decursivo do cuidado, não relacionado à patologia de base, o qual resulta em dano desnecessário ao paciente. Estima-se que os EA tenham incidência de 4 a $16 \%$ entre todos os pacientes hospitalizados. Aproximadamente, $48 \%$ de todos os EA envolvem a atividade cirúrgica, com evitabilidade em 30 a $50 \%$ dos $\operatorname{casos}^{(1-2)}$.

Os EA cirúrgicos apresentam grande potencial para ocasionar danos graves, podem levar à morte, trazer repercussões físicas e emocionais para o paciente e sua família, aumentar o tempo de internação, elevar os custos hospitalares e, consequentemente, gerar um impacto negativo para a instituição, uma vez que sua ocorrência está diretamente relacionada à qualidade da assistência à saúde ${ }^{(3-4)}$.

Nesse contexto, em outubro de 2004, a OMS criou a "Aliança Mundial para a Segurança do Paciente", com o objetivo de reduzir os danos desnecessários ou potenciais associados aos cuidados de saúde e promover o desenvolvimento de políticas e estratégias na atenção à saúde ${ }^{(2)}$. Para atingir metas relacionadas à segurança em cirurgia, a OMS lançou, no ano de 2008, o manual de orientação "Cirurgia Segura Salva Vidas", recomendando a utilização de uma lista de verificação (checklist) de segurança em cirurgia. Esse manual foi adaptado no Brasil pela Agência Nacional de Vigilância Sanitária (ANVISA) indicando padrões que devem ser aplicados nas instituições de saúde para melhorar a segurança na assistência cirúrgica ${ }^{(4-5)}$.

O checklist foi desenvolvido a partir de revisões de práticas baseadas em evidências que identificaram as causas mais comuns de danos ao paciente no período perioperatório e pode ser empregado em qualquer instituição de assistência à saúde. Tem como objetivo reduzir a ocorrência de danos ao paciente, auxiliando as equipes cirúrgicas a seguirem de forma sistemática passos críticos de segurança, configurando-se como uma barreira para evitar falhas humanas, buscando padronizar as atividades que deverão ser realizadas ${ }^{(4,6)}$.

Estudos demonstram que a utilização do checklist também facilita a coordenação da equipe cirúrgica, reduz as taxas de mortalidade e complicações em pacientes cirúrgicos, aumenta a adesão à antibioticoprofilaxia e reduz o número de erros por falha de comunicação entre a equipe $^{(5,7)}$.

Para que o checklist seja devidamente aplicado, faz-se necessário que a equipe que atua no centro cirúrgico $(\mathrm{CC})$ reconheça a importância desse instrumento, tenha conhecimento sobre cada uma de suas etapas e esteja envolvida com a sua implantação. Orienta-se que um único e qualquer membro da equipe que participe do procedimento cirúrgico seja responsável pela sua aplicação ${ }^{(8)}$. Um ponto facilitador em ter um profissional de enfermagem como responsável pela aplicação do instrumento, em especial os técnicos de enfermagem (TE) que atuam como circulante de sala, é a sua capacidade de se mover em todas as etapas no cuidado do paciente durante o período perioperatório. Contudo, é necessário que esse profissional tenha conhecimento e esteja apto a utilizar o instrumento ${ }^{(9)}$.

Considerando a importância da adesão ao checklist para a redução dos EA cirúrgicos, o presente estudo traz como objetivo compreender o conhecimento e práticas dos técnicos de enfermagem que atuam como circulantes nas salas de cirurgia sobre cirurgia segura e a aplicação do checklist em um hospital de ensino da Zona Da Mata Mineira, Brasil.

\section{MÉTODOS}

Trata-se de um estudo de natureza qualitativa. Esse tipo de pesquisa permite o aprofundamento relativo aos significados que as pessoas atribuem às suas ações e relações humanas, objetivando uma abordagem compreensiva dos sujeitos que vivenciam uma dada experiência. Contribui para a melhor compreensão da distância entre o conhecimento e a prática, na medida em que auxilia na elucidação dos sentimentos das pessoas, 
explicitando suas ações diante de um problema em situação ${ }^{(10)}$.

O presente estudo foi realizado no Centro Cirúrgico de um hospital de médio porte da Zona da Mata Mineira, considerado de natureza filantrópica, de caráter assistencial e que tem por finalidade a prestação de assistência médicohospitalar. A referida instituição é vinculada como hospital de ensino da Universidade Federal de Viçosa, onde os alunos desenvolvem atividades práticas. $\mathrm{O}$ checklist de cirurgia segura é um instrumento que se baseia na conferência de vários itens relevantes para minimizar a ocorrência de erro e foi implantado no CC da instituição onde se realizou este estudo, no ano de 2014, para todas as especialidades cirúrgicas. Por ser um CC pequeno e com número reduzido de profissionais, o enfermeiro coordenador do setor orientou os circulantes de sala individualmente em relação ao uso do checklist

Os participantes da pesquisa foram dez TE que atuam como circulantes de sala no CC. Os critérios de inclusão foram: ser TE do CC, atuar como circulante da sala operatória independentemente do tempo de exercício na função. Os critérios de exclusão foram: não ser $T E$, ser $T E$ que trabalha no setor durante $O$ período noturno e estar afastado do cargo por qualquer motivo. Os TE que atuam no período noturno desenvolvem prioritariamente atividades relacionadas à previsão e provisão de materiais para as cirurgias do dia seguinte, motivo pelo qual foram excluídos do estudo.

A coleta de dados foi realizada no mês de setembro de 2016, por meio de técnica de entrevista semiestruturada conduzida por meio de um roteiro contendo cinco perguntas abertas, as quais buscavam compreender a concepção dos TE sobre cirurgia segura bem como as estratégias utilizadas pela equipe cirúrgica para a prevenção do erro envolvendo a assistência ao paciente cirúrgico. Buscou-se também informações referentes ao uso do checklist na prática cotidiana, além dos aspectos que favorecem e/ou dificultam a aplicação efetiva desse instrumento.

As entrevistas ocorreram dentro do próprio setor, em uma sala reservada para o participante expor com tranquilidade e segurança as suas vivências. Os depoimentos foram gravados mediante autorização dos participantes do estudo e posteriormente transcritos na íntegra. Para fins de preservação do anonimato, os TE foram referidos pela letra E (entrevistado), sucedido do número correspondente à ordem em que forem realizadas as entrevistas, a saber: E1, E2, E3 e assim sucessivamente.

Para analisar os dados obtidos, foi utilizada a técnica de Análise de Conteúdo ${ }^{(11)}$. Para tanto, foram realizadas leituras criteriosas de cada depoimento, com o objetivo de possibilitar uma compreensão geral acerca das respostas obtidas. Os depoimentos foram analisados a partir de eixos temáticos, com o intuito de investigar tendências e confluências de conceitos, com posterior realização da categorização. Posteriormente foi realizada a interpretação dos dados de acordo com a literatura pertinente à temática.

A pesquisa foi autorizada pelo Comitê de Ética do hospital onde foi realizado o estudo mediante assinatura do termo de anuência, e, também pelo Comitê de Ética em Pesquisa com Seres Humanos sob o protocolo $\mathrm{n}$ 57938016.0.0000.5153, conforme os princípios éticos inscritos na Resolução 466/12 do Conselho Nacional de Saúde.

\section{RESULTADOS E DISCUSSÃO}

Em relação às características dos participantes do estudo, verificou-se que dentre os dez entrevistados, sete eram do sexo feminino. O participante mais jovem tinha 22 anos e o mais velho 46 , a média de idade entre eles era de 30 anos. O tempo de formação dos TE variou de um ano e sete meses a 12 anos e o tempo de atuação no CC foi de um mês a 10 anos.

Após leitura, análise e interpretação dos depoimentos, os dados foram organizados em três categorias: "Cirurgia segura na concepção dos TE", "A prevenção do erro em CC como sinônimo de identificação do paciente e atenção aos parâmetros clínicos" e "Compreendendo o uso do checklist de cirurgia segura: dissonâncias teórico-práticas".

\section{Cirurgia segura na concepção dos TE}

Entende-se que cirurgia segura é aquela na qual são adotadas medidas para redução de complicações e mortalidade associadas à cirurgia, ocorridas no transoperatório, seja antes, durante ou após a realização de procedimentos cirúrgicos $^{(12)}$.

Os depoimentos a seguir ilustram o que é uma cirurgia segura na perspectiva dos TE:

"[...] o técnico tá ali fazendo ... a parte dele certinha [...] o anestesista, o cirurgião [...] o material estando tudo esterilizado [...] instrumental, carrinho funcionando [...]" (E3). 
"[...] Garantir a segurança do paciente desde uma sala bem limpa, até o material também que vai ser usado na cirurgia [...] totalmente estéril, corretamente estéril [...] uma assepsia correta do local que vai ser feita a cirurgia, uso dos métodos que previnem os riscos de infecções [...] e fazer a assepsia das mãos direitinho" (E4).

"[...] limpeza, e equipe completa, material estéril [...]" (E8).

Os relatos permitem identificar uma preocupação importante em relação à esterilização dos materiais e limpeza da sala, as quais são medidas para redução do risco de infecção associada ao procedimento operatório. Para garantir a segurança operatória, é necessária a utilização de métodos para evitar a Infecção do Sítio Cirúrgico $(\mathrm{ISC})^{(2)}$, que é a causa mais frequente de graves complicações chegando a atingir 38\% dos pacientes cirúrgicos, além de contribuir para o aumento da morbimortalidade, do período de internação e dos custos hospitalares ${ }^{(13)}$.

A ISC pode ser considerada um EA grave, que depende da possibilidade de contaminação da incisão operatória durante o procedimento no qual há multiplicação de microrganismos na ferida cirúrgica. Pode acometer órgãos e cavidades manipuladas durante o ato operatório com ocorrência de até trinta dias após a data da cirurgia ${ }^{(13-15)}$.

Para reduzir as taxas de ISC, faz-se necessária a sistematização do processamento de instrumentais (desde a limpeza até o armazenamento), a utilização de medidas que comprovem a eficácia da esterilização de campos, instrumentos e kits operatórios (como monitorização química e biológica em autoclaves), bem como a higienização das mãos pela equipe, uso de máscara, capotes e parâmetros protetores, uma correta assepsia do local onde será realizada a incisão e manuseio de forma asséptica dos materiais utilizados para a realização do procedimento operatório, entre outras medidas $^{(15-16)}$.

Outras medidas importantes para a segurança do paciente, associadas ao ato anestésico-cirúrgico, foram mencionadas pelos TE como: aferição dos dados vitais, equipamentos necessários ao procedimento, monitorização do paciente, paciente certo e apresentação do risco cirúrgico.
Nessa categoria, observa-se que os depoimentos dos TE apresentam um conceito incipiente do que é cirurgia segura, concentrando-se em aspectos relacionados à limpeza do ambiente e provisão de materiais corretamente esterilizados. Medidas importantes para evitar complicações cirúrgicas como demarcação do sítio cirúrgico e lateralidade, comunicação eficaz entre a equipe, escrita legível, avaliação do risco de grandes perdas sanguíneas, contagem de compressas e instrumentais, entre outros $^{(2,12)}$ não foram citadas nos depoimentos.

Dessa forma, percebeu-se que, apesar dos participantes apontarem algumas medidas de segurança, não conseguem defini-las de acordo com conceito de cirurgia segura. Para eles, uma cirurgia segura é aquela em que são seguidas rigorosamente as normas e rotinas adotadas pela instituição, demonstrando assim não ter conhecimento cientificamente fundamentado sobre questões relacionadas à segurança cirúrgica. A falta de conhecimentos sobre segurança pode levar a inúmeros prejuízos ao paciente, principalmente no $\mathrm{CC}$, visto que esse setor realiza procedimentos de alta e média complexidades, susceptíveis à ocorrência de danos irreparáveis.

\section{A prevenção do erro em CC como sinônimo de identificação do paciente e atenção aos parâmetros clínicos}

Existe uma tênue interface entre o EA e o erro, a frequência destes é considerada como um indicador de qualidade da assistência e é um dos pontos de avaliação em alguns processos de acreditação $^{(3)}$. O erro pode ser caracterizado como um incidente de natureza intencional ou não, podendo resultar em danos ao paciente. No CC, os erros podem ser relacionados aos equipamentos e insumos, como ausência de manutenção e falha na previsão e provisão de recursos materiais ou podem estar associados à desatenção na assistência, podendo causar danos diretos ao paciente, como quedas da mesa cirúrgica e extubações não programadas ${ }^{(17)}$.

As falas abaixo ilustram como os TE identificam no dia a dia a prevenção da ocorrência do erro envolvendo o paciente cirúrgico.

"[...] se aconteceu qualquer erro voltar e falar com superior: aconteceu tal erro, fiz tal medicação [...] errar infelizmente a gente está suposto a errar, agora basta a gente ter 
humildade e responsabilidade pra voltar atrás e falar que fez o erro" (E1).

"[...] você ficar atento aos dados vitais [...]"

“[...] eu acho que é primordial a questão de identificação [...] de tudo, seja lá do paciente, daquilo que você for administrar no paciente, daquilo que o paciente for fazer [...]" (E8).

Em consonância com os relatos dos entrevistados, ficou evidente que eles não souberam citar medidas que possam, cientificamente, serem reconhecidas para prevenir o erro. No entanto, os TE reconhecem a importância de relatar o erro ao coordenador. Esse fato pode sugerir que a cultura de punição começa a dar lugar à cultura de segurança, pois quando a equipe reporta às ocorrências, torna-se mais fácil a aplicação de medidas eficazes para a prevenção de possíveis erros futuros ${ }^{(18)}$.

Mesmo alegando que comunicam o erro, muitos profissionais apresentam dificuldades para expor erros relacionados à assistência por falta de abertura com a equipe para a discussão dessas falhas. Outros afirmam ainda não perceberem a ocorrência do erro em circunstâncias nas quais se sintam tensos ou hostilizados e que têm dificuldades para se expressarem, o que é um ponto negativo em relação à segurança do paciente ${ }^{(19)}$.

Alguns fatores como a insuficiência de profissionais, sobrecarga de trabalho, dificuldades no relacionamento com a equipe e falta de supervisão pela enfermagem são reconhecidas como possíveis causas que podem levar à ocorrência do erro ${ }^{(17-19)}$.

Um dos participantes referiu-se à questão da identificação do paciente como uma questão importante relacionada à segurança. Uma das metas internacionais relacionadas à segurança do paciente é a identificação correta, no intuito de reduzir o número de cirurgias realizadas no paciente errado ${ }^{(7)}$.

A falta identificação do paciente foi apontada como um importante fator de risco que leva a insegurança, a qual pode acarretar em danos severos ao paciente ${ }^{(18)}$. A identificação do paciente é de responsabilidade multidisciplinar e quando realizada corretamente previne diversos erros, como: erro de medicação, procedimentos ou cirurgias em paciente e locais errados, erros de diagnósticos, dentre outros ${ }^{(20)}$.

A capacitação profissional, outro item citado somente uma vez, porém não menos importante, também emergiu da fala de um dos entrevistados. O profissional que atua no CC deve estar "habilitado tecnicamente ${ }^{(18) "}$ para evitar falhas e, consequentemente, reduzir o número de erros.

"[...] A pessoa estar preparada para aquilo que vai fazer [...] saber o que tá fazendo e fazer correto [...]" (E2).

Em nenhum momento, os TE citaram o uso do instrumento como uma forma de prevenção do erro em CC. Esse fato evidencia mais uma vez que os TE demonstraram não conhecer por completo questões relacionadas às práticas de segurança em procedimentos cirúrgicos e que, apesar de fazer o uso do instrumento, não sabem que este foi desenvolvido para minimizar a ocorrência de erros. A forma de implantação do instrumento no setor sem treinamento sistemático e sensibilização da equipe envolvida pode ter contribuído.

A falta de capacitações e o treinamento de profissionais que atuam na assistência cirúrgica já foi mencionada como um problema para a segurança do paciente cirúrgico em países em desenvolvimento ${ }^{(2)}$. Além disso, a implementação de qualquer instrumento requer a capacitação dos profissionais que irão utilizá-lo, bem como o envolvimento de toda a equipe de saúde na sua implantação, para que estes possam compreender o porquê de estarem utilizando uma nova ferramenta, quais os benefícios ela trará para a qualidade de assistência, além de possibilitar que o profissional tenha um olhar crítico-reflexivo em relação às suas práticas em saúde ${ }^{(21-22)}$

\section{Compreendendo o uso do checklist de cirurgia segura: dissonâncias teórico-práticas}

O checklist desenvolvido pela OMS busca padronizar as atividades que deverão ser realizadas na sala de cirurgia, auxiliando na memorização de suas etapas, a fim de melhorar o desempenho dos profissionais ${ }^{(7)}$. É constituído por 19 itens e toda equipe cirúrgica participa da sua checagem, inclusive o paciente. Há uma orientação sequencial e sistematizada para aplicá-lo em três momentos: antes da indução anestésica em que são checadas algumas informações com o paciente; antes da incisão cirúrgica quando é realizada a leitura dos itens do checklist em voz alta e antes da saída do paciente da sala de cirurgia para conferência dos materiais utilizados no procedimento, assegurando que nenhum deles ficou retido no paciente $\mathrm{e}^{(3,7,8)}$. 
Quando os TE foram indagados se o paciente ou algum outro profissional da equipe fornece informações para o preenchimento do checklist, surgiram os seguintes relatos:

"Eu nunca olho com outra pessoa ou com o médico. Sempre olho o prontuário" (E1).

"Sim, porque se eu pegar a cirurgia pela metade de um colega que vai sair então ele vai me passar [...]" (E5).

"Sim. O Paciente ele já diz qual cirurgia ele vai fazer, onde ele vai fazer, se ele tiver lúcido [...]" (E7).

Percebe-se pelos relatos, que os TE não estão sabendo utilizar o instrumento de forma sistemática conforme orientado pela OMS. Os depoimentos sugerem que o checklist tem sido preenchido como um documento qualquer obrigatório no prontuário, sem a devida importância na ordem de preenchimento. Segundo orientações da OMS, o checklist deve ser aplicado por um único membro da equipe, do início ao fim do procedimento. Além disso, se for detectada alguma falha, deve-se interromper a verificação até que esta seja resolvida ${ }^{(2)}$.

Apesar de alguns TE relatarem que fazem perguntas ao paciente, ficou evidente que eles não utilizam o checklist de forma correta, não realizam a checagem nos três momentos e preenchem sem a participação do anestesiologista e do cirurgião, não estando, portanto, em conformidade com o preconizado pela OMS e ANVISA.

O uso inadequado do instrumento abre lacunas na assistência que propicia falhas à segurança. Um estudo realizado no Rio Grande do Norte, cujo objetivo foi avaliar a adesão ao checklist em cirurgias urológicas e ginecológicas, revelou que as etapas antes da indução anestésica e incisão cirúrgica são as que apresentam maiores problemas em relação à adesão ao checklist. Além disso, o estudo identifica a necessidade de demonstração da finalidade e do preenchimento correto do instrumento antes e durante a sua implantação ${ }^{(5)}$.

Outra pesquisa realizada no sul do Brasil também identificou resultados semelhantes. Verificou-se que a checagem não acontece verbalmente e que a adesão ao instrumento em relação à identificação do paciente, procedimento a ser realizado, confirmação da equipe e da contagem de materiais não se deu conforme preconizado ${ }^{(23)}$.

Apesar de não utilizarem o instrumento de forma apropriada, os TE consideram que 0 checklist é importante para a continuidade do cuidado, pois nele está registrado o que aconteceu durante a cirurgia (medicamentos utilizados, procedimentos invasivos, entre outros), oferecendo informações importantes para quem for receber o paciente no pósoperatório. Os depoimentos a seguir ilustram o exposto:

"[...] ali fica registrado ele indo lá pra fora [...] outras pessoas vão ter acesso, aí vai saber o percurso do paciente aqui dentro do CC [...]" (E4).

"[...] quando já passar o paciente daqui lá para o outro setor que ele vai ficar internado vai dar pra ele saber. Eles vão olhar ali e vão ver qual foi o tipo de cirurgia que fez, se tá com sonda $[\ldots] "$ (E6).

"[...] o checkist ele te obriga você a ter uma identificação do paciente [...] as perguntas que ficam contidas nele que te dá um certo respaldo de alguma coisa que vai te levar ao [...] já é meio caminho andado" (E8).

Alguns TE relataram que o checklist evita o erro, pois obriga o profissional a identificar o paciente, outros reconhecem que o instrumento orienta as ações durante a cirurgia. No entanto, nenhum deles demonstrou conhecimento mais aprofundado em relação às concepções teóricas que envolvem a segurança cirúrgica e o uso do checklist.

Nenhum dos depoimentos faz referência ao checklist como uma ferramenta desenvolvida e recomendada pela a OMS para melhorar a segurança na assistência cirúrgica, sugerindo que o instrumento foi implantado sem contextualização com temas relacionados à segurança do paciente e mundialmente discutidos. Analisando as falas de forma reflexiva, percebe-se que os TE concebem o checklist como apenas mais um documento que deve ser preenchido, não o compreendendo como uma ferramenta estratégica que faz parte de um programa desenvolvido com o objetivo de elevar os padrões de segurança na assistência à saúde.

Os profissionais de enfermagem em CC percebem a necessidade de garantir a segurança do paciente. No entanto, é essencial sensibilizar a equipe quanto à importância do checklist como uma ferramenta útil para melhorar a segurança do paciente, antecipando eventuais danos e promovendo a qualidade da assistência. Além disso, a utilização do checklist também qualifica o trabalho da equipe envolvida no procedimento cirúrgico, centralizando o cuidado no paciente ${ }^{(24)}$. 
A maioria dos TE concordou ao dizer que o checklist favorece a assistência, pois nele se encontram as informações referentes ao período intraoperatório e que serão importantes para a continuidade do cuidado. Apenas três entrevistados apontaram a falta de tempo como dificuldade para o preenchimento do checklist. Porém, estima-se que é necessário o tempo total de três minutos para aplicação das três fases do processo de verificação ${ }^{(5,8)}$. Um dos entrevistados pontuou que o checklist contém algumas informações irrelevantes. Vale ressaltar que a $\mathrm{OMS}^{(14)}$ orienta e indica mudanças na estrutura do checklist de acordo com a realidade de cada instituição, uma vez que no estabelecimento onde foi realizado este estudo o instrumento já foi adaptado de acordo com a realidade da instituição.

\section{CONSIDERAÇÕES FINAIS}

$O$ estudo evidenciou que os TE demonstraram não ter conhecimentos cientificamente fundamentados sobre os aspectos que envolvem a segurança cirúrgica. Apesar do checklist de cirurgia segura estar implantado na instituição em que atuam, os TE têm dificuldade de falar acerca da lista de verificação, não reconhecem o instrumento como ferramenta de prevenção/redução dos erros comumente ocorridos em CC e não sabem utilizála de maneira correta, seguindo os passos críticos conforme preconizado.

Para que o checklist seja usado da forma correta e atinja seu objetivo é necessário que as instituições de saúde adotem a cultura de segurança e que haja treinamento e capacitação dos profissionais para utilizá-lo. É preciso que os profissionais de saúde vejam essa ferramenta não só como mais um documento a ser preenchido, mas como uma estratégia que irá diminuir a ocorrência do erro no $\mathrm{CC}$ e, consequentemente, melhorar a qualidade da assistência prestada ao paciente cirúrgico.

O estudo apresenta limitações por retratar a realidade de um único cenário de estudo, com características particulares, não possibilitando a generalização dos resultados aqui encontrados. Apesar disso, o estudo traz contribuições importantes ao elucidar como o checklist tem sido utilizado na prática cirúrgica apontando para o desafio de transformar um documento obrigatório de checagem em instrumento necessário e capaz de melhorar a segurança do paciente cirúrgico.

\section{REFERÊNCIAS}

1 Moura MLO, Mendes W. Avaliação de eventos adversos cirúrgicos em hospitais do Rio de Janeiro. Rev Bras Epidemiol. 2012;15(3):523-34. DOI: 10.1590/S1415-790X2012000300 007

2 Organização Mundial da Saúde (OMS). Segundo desafio global para a segurança do paciente: Cirurgias seguras salvam vidas. Rio de Janeiro: Organização Pan-Americana da Saúde; 2009.

3 Vendramini RCR, Silva EA, Ferreira KASL, Possari JF, Mollo WRB. Segurança do paciente em cirurgia oncológica: Experiência do Instituto do Câncer do Estado de São Paulo. Rev Esc Enferm USP. 2010;44(3):827-32. DOI: $10.1590 / 50080-$ 62342010000300039

4 Santana HN, Costa MMM, Oliveira DCAN, Gomes SM, Sousa FC, Santos ACRB, et al. A segurança do paciente cirúrgico na perspectiva da vigilância sanitária - uma reflexão teórica. Vig Sanit Debate 2014;2(2):34-42. DOI: 10.3395/vd.v2i2.124

5 Freitas MR, Antunes AG, Lopes BNA, Fernandes FC, Monte LC, Gama ZAS. Avaliação da adesão ao checklist de cirurgia segura da OMS em cirurgias urológicas e ginecológicas, em dois hospitais de ensino de Natal, Rio Grande do Norte, Brasil. Cad Saúde Pública 2014;30(1):137-48. DOI: 10.1590/ 0102-311X00184612

6 Pires MPO, Pedreira MLG, Peterlini MAS. Cirurgia segura em pediatria: Elaboração e validação de checklist de intervenções préoperatórias. Rev Latino-Am Enfermagem 2013 [citado em 4 jan 2018]; 21(5):[08 telas]. Disponível em: http://www.scielo.br/pdf/rlae/v21n5/pt $010 \quad 4-$ 1169-rlae-21-05-1080.pdf

7 Bohomol E, Tartali JA. Eventos adversos em pacientes cirúrgicos: Conhecimento dos profissionais de enfermagem. Acta Paul Enferm. 2013;26(4):376-81. DOI: 10.1590/S0103-21002013 000400012

8 Pancieri AP, Santos BP, Avila MAG, Braga EM. Checklist de cirurgia segura: Análise da segurança e comunicação das equipes de um hospital escola. Rev Gaúch Enferm. 2013;34(1):71-8. DOI: 10.1590/S1983-14472013000100009

9 Monteiro F, Silva LR. "Checklist" lista de verificação de segurança cirúrgica: Avaliação e intervenção. Rev Ciênc Méd Biol. 2013;12(4):4825. DOI: $10.9771 / \mathrm{cmbio.v12i4.9196}$

10 Minayo MCS. Análise qualitativa: Teoria, passos e fidedignidade. Ciênc Saúde Coletiva 2012;17(3):621-6. DOI: 10.1590/S1413-81232012 $\underline{000300007}$ 
11 Santos FM. Análise de conteúdo: A visão de Laurence Bardin. REVEDUC. 2012;6(1):383-7. DOI: 10.14244/\%2519827199291

12 Muniz RV, Bohrer BBA, Mulazzani MP, Erig LS, Santos SM, Santos HB. Criação e evolução da lista de verificação de cirurgia segura específica para oftalmologia. Rev ACRED. 2015 [citado em 4 jan 2018]; 5(9):85-101. Disponível em: https://dialnet. unirioja.es/servlet/articulo?codigo $=5626618$ 13 Oliveira AC, Gama CS. Avaliação da adesão às medidas para a prevenção de infecções do sítio cirúrgico pela equipe cirúrgica. Rev Esc Enferm USP. 2015;49(5):767-74. DOI: 10.1590/S0080$\underline{623420150000500009}$

14 Gebrim CFL, Rodrigues JG, Queiroz MNR, Barreto RASS, Palos MAP. Análise da profilaxia antimicrobiana para a prevenção da infecção do sítio cirúrgico em um hospital do Centro-Oeste Brasileiro. Cienc Enferm. 2014 [citado em 4 jan 2018]; 20(2):103-15. Disponível em: http://www. scielo.cl/pdf/cienf/v20n2/art 11.pdf

15 Aguiar APL, Prado PR, Opitz SP, Vasconcelos SP, Faro ARMC. Fatores associados à infecção de sítio cirúrgico em um hospital na Amazônia Ocidental Brasileira. Rev SOBECC 2012 [citado em 4 jan 2018]; 17(3):60-70. Disponível em: http://www.sobecc. org.br/arquivos/artigos/uploads/snf/arquivos/An o17 n3 \%20jul set2012 (Fatores associados inf eccao de sitio cirurgico em um hospital na a mazonia ocidental brasileira).pdf

16 Sociedade Brasileira de Enfermeiros de Centro Cirúrgico Recuperação Anestésica e Centro De Material e Esterilização (SOBECC). Práticas recomendadas da SOBECC. 6a ed. São Paulo: SOBECC; 2013.

17 Duarte SCM, Stipp MAC, Silva MM, Oliveira FT. Eventos adversos e segurança na assistência de enfermagem. Rev Bras Enferm. 2015;68(1):14454. DOI: 10.1590/0034-7167.2015680120p

18 Souza FT, Garcia MC, Rangel PPS, Rocha PK. Percepção da enfermagem sobre os fatores de risco que envolvem a segurança do paciente pediátrico. Rev Enferm UFSM. 2014;4(1):152-62. DOI: $10.5902 / 217976928781$

19 Correggio TC, Amante LN, Barbosa SFF. Avaliação da cultura de segurança do paciente em Centro Cirúrgico. Rev SOBECC. 2014;19(2):67-73. DOI: $10.4322 /$ sobecc. 2014.012

20 World Health Organization (WHO). Conceptual framework for the international classification for patient safety: Verson 1.1. Genève: WHO; 2009. 21 Silva CSO, Pinheiro GO, Freitas BC, Figueiredo BM, Macedo WTP, Silva EC. Checklist de cirurgia segura: Os desafios da implantação e adesão nas instituições hospitalares brasileiras. Rev. Espaço ciência e saúde. 2017 [citado em 8 abr 2017]; 5(2):72-86. Disponível em: file:///C:/Users/N\%C3\% BAbia/Downloads/546717198-1-PB.pdf

22 Sandlhofer G, Mosbacher N, Karina L, Kober B, Jantscher L, Berghold A, et al. Implementation of a surgical safety checklist: Interventions to optimize the process and hints to increase compliance. PLoS One 2015;10(2):1-14. DOI: 10.1371/journal.pone.0116926

23 Maziero ECS, Silva AEBC, Mantovani MF, Cruz EDA. Adesão ao uso de um checklist cirúrgico para segurança do paciente. Rev Gaúcha Enferm. 2015;36(4):14-20. DOI: 10.1590/1983-1447.2015. $\underline{04.53716}$

24 Gomes CDPP, Santos AA, Machado ME, Treviso $P$. Percepção de uma equipe de enfermagem sobre a utilização do checklist cirúrgico. Rev SOBECC. 2016;21(3):140-5. DOI: 10.5327/Z14144425201600030004

Nota: Pesquisa originada de um Trabalho Conclusão de Curso apresentado ao Departamento de Medicina e Enfermagem da Universidade Federal de Viçosa, para aquisição do título de bacharel em enfermagem.

Recebido em: 10/11/2017

Aprovado em: 19/11/2018

Endereço de correspondência:

Núbia da Conceição Santos Ferreira Rua Conceição, 264 - Bairro de Fátima CEP: 36572200 - Viçosa/MG - Brasil

E-mail: nubiafefg@gmail.com 